\title{
Protective effect of FOXP3-mediated miR-146b-5p/Robo1/NF-кB system on lipopolysaccharide-induced acute lung injury in mice
}

\author{
Jiang Zhu' ${ }^{1}$, Gaoli Chen ${ }^{2}$ \\ ${ }^{1}$ Department of Respiratory and Critical Care Medicine, Sichuan Academy of Medical Sciences \& Sichuan Provincial People's Hospital, University \\ Hospital of Electronic Science \& Technology of China, Chengdu, China; ${ }^{2}$ Department of Blood Transfusion, Teaching Hospital of Chengdu \\ University of TCM, Chengdu, China \\ Contributions: (I) Conception and design: All authors; (II) Administrative support: All authors; (III) Provision of study materials or patients: All \\ authors; (IV) Collection and assembly of data: All authors; (V) Data analysis and interpretation: All authors; (VI) Manuscript writing: All authors; (VII) \\ Final approval of manuscript: All authors. \\ Correspondence to: Gaoli Chen. Department of Blood Transfusion, Teaching Hospital of Chengdu University of TCM, No.39, Shierqiao Road, Jinniu \\ District, Chengdu 610072, China. Email: chengaoli2020@163.com.
}

Background: As a key transcription factor, forkhead box protein 3 (FOXP3) plays an important role in the development and function of natural cluster of differentiation 4 [CD4 (+)] regulatory T cells (Treg cells). However, the function of FOXP3 in Lipopolysaccharide (LPS)-induced acute lung injury (ALI) through regulating miR-146b-5p is unclear. This research aimed to disclose the regulatory effect of the FOXP3mediated miR-146b-5p/Roundabout 1 (Robo1)/NF- $\kappa$ B system on LPS-induced ALI in mice.

Methods: The mice were subjected to $5 \mathrm{mg} / \mathrm{kg}$ of LPS via intratracheal instillation to induce ALI and generate the ALI model. Mice was divided into five group, including control group, ALI group, ALI + FOXP3 group, the ALI + miR antagomir group and ALI + miR antagomir+ FOXP3 group. Lung tissue injury were detected by hematoxylin and eosin (HE) staining. Lung wet/dry weight ratio, total cells in bronchoalveolar lavage fluid (BALF), total protein in BALF and the polymorphonuclear leukocyte (PMN) in BALF were detected. The levels of tumor necrosis factor- $\alpha$ (TNF- $\alpha$ ), Interleukin 6 (IL- 6 ) and IL- $1 \beta$ were detected by enzyme-linked immunosorbent assay (ELISA) kit. The dual-luciferase reporter assay were used to detect the target relationship between FOXP3 and Robo1. Mice was divided into five group, including control group, ALI group, ALI + FOXP3 group, ALI + Robo1 group and ALI + FOXP3 + Robo1 group. The protein levels of FOXP3, Robo1 and p-p65 were detected by western bolt. The mRNA levels of miR-146b$5 \mathrm{p}$ and Robol were detected by quantitative reverse transcription polymerase chain reaction (qRT-PCR).

Results: Although protein expression levels of FOXP3 were significantly down-regulated in the ALI model, the increased FOXP3 levels promoted an increase in miR-146b-5p. Compared with the control group, the ALI model group exhibited severe histopathologic injury, such as thickening of the alveolar wall, pulmonary congestion, and decreased alveolar numbers. By mediating the overexpression of miR146b-5p, FOXP3 also increased alveolar clearance and inhibited inflammatory responses in the ALI model. Importantly, Robo1 is a potential target of miR-146b-5p.

Conclusions: FOXP3 could inhibit NF- $\kappa \mathrm{B}$ activation, reduce lung pathological damage, and inhibit inflammatory responses by mediating the miR-146b-5p/Robo1/NF- $\kappa \mathrm{B}$ system in the ALI model. These results may provide a new potential target for the treatment of ALI disease.

Keywords: FOXP3; LPS-induced acute lung injury; miR-146b-5p/Robo1/NF-кB

Submitted Oct 16, 2020. Accepted for publication Dec 13, 2020.

doi: $10.21037 / \mathrm{atm}-20-7703$

View this article at: http://dx.doi.org/10.21037/atm-20-7703

(c) Annals of Translational Medicine. All rights reserved. 


\section{Introduction}

Acute respiratory distress syndrome (ARDS) is a clinically important complication of severe acute lung injury (ALI). Infections such as sepsis and pneumonia are the leading causes of ALI/ARDS $(1,2)$, and histology shows the pulmonary manifestations of an acute systemic inflammatory process characterized by pulmonary infiltrates, hypoxaemia, and oedema. As many as 25 cases per 100,000 are reported annually, with a high prevalence in young people $(3,4)$. The Lipopolysaccharide (LPS)-induced ALI model is often used to examine lung injury and many components of its response, particularly the acute pathways. At the same time, LPS is an effective activator of the innate immune pathway, greatly imitating the pathological changes of $\operatorname{ALI}(5,6)$.

Regulatory $\mathrm{T}$ (Treg) cell subsets have specific transcription fork head box protein 3 (FOXP3), a unique cell type that maintains immune homeostasis by controlling the response of effector T (Teff) cells. Research has shown that FOXP3 is also called fork head/winged helix transcription factor (FOXP3). FOXP3 is specifically expressed on cluster of differentiation (CD)4+CD25+ regulatory $\mathrm{T}$ cells and is a key transcription factor for its production and development (7). The regulation and proliferation rate of lung epithelial cells following lung injury is strongly correlated with the number of FOXP3 + Treg CD103 (8). One report indicates that FOXP3 expression in Tregs may be down-regulated in the inflammatory alveolar microenvironment by LPS-induced ALI (9). This raises the question of whether FOXP3 plays a protective role in LPS-induced ALI models.

Diverse biological processes have been associated with miRNAs, such as inducing M2 polarization (10), regulating insulin secretion (11), and suppressing cancer invasion $(12,13)$. Further, miR146 has been associated with the control of inflammatory responses (14), inducing the differentiation of macrophages into M2 cells (15), inhibiting cancer cell metastasis (16), and brake myeloproliferation (17). It is increasingly recognized that the human genome contains two miR-146 genes on chromosomes 5 and 10, respectively, including miR-146a (18) and miR-146b (19). Inflammation caused LPS-induced injury in A549 and H1975 cells and miR146 has been shown to relieve apoptosis (20). Importantly, FOXP3-induced mir-146 a/b has been displayed to not only restrain breast cancer cell proliferation and promote apoptosis, but also reversely regulate activation of $\mathrm{NF}-\kappa \mathrm{B}$ by inhibiting the expression of tumors necrosis factor receptor related factor 6 (TRAF6) and receptorrelated kinases (IRAK1) (21).

Previous research has exhibited that miR-146b-5p protects oligodendrocyte precursor cells from oxygen/ glucose deprivation-induced injury through regulating Keap1/Nrf2 signaling via targeting bromodomaincontaining protein 4 (22). MiR-146b-5p attenuates the inflammatory response of glomerular mesangial cells by inhibiting the expressions levels of TRAF6 and IRAK1 in lupus nephritis (23). p16INK4a inhibits the proliferation of osteosarcoma cells through regulating the miR-146b-5p/ TRAF6 pathway (24). Reduced miR-146b-5p expression in T-ALL may lead to the up-regulation of IL-17A, which then promotes $\mathrm{T}$ cell acute lymphoblastic leukemia cell migration and invasion by up-regulating matrix metallopeptidase-9 (MMP9) via NF- $\mathrm{KB}$ signaling (25). Slit homolog 2 (Slit2)/Robo1 signaling is involved in angiogenesis of glomerular endothelial cells exposed to a diabetic-like environment (26). Robol promotes angiogenesis in hepatocellular carcinoma through the Rho family of guanosine triphosphatases' signaling pathway (27). miR-490-5p inhibits cell proliferation, migration and invasion, but promoted cell apoptosis of Hep3B cells by inhibiting Robo1 (28). miR-365 promotes lung carcinogenesis by downregulating the ubiquitin-specific protease 33 (USP33)/Slit2/Robo1 signalling pathway (29).

The activation of NF- $\kappa \mathrm{B}$ can be induced by LPS binding to Toll-like receptor 4 (TLR4) (30). This activation can regulate a range of gene expressions, including TNF- $\alpha$, IL- $1 \beta$, and IL-6 (31), which are cytokines that injure lung tissue (32). In addition, NF- $\mathrm{KB}$ is the key to the maximum expression of multiple cytokines in the pathogenesis of ALI (33). Research has shown that IL-22 can decrease FOXP3 expression by activating NF- $\kappa \mathrm{B}$ (34) and that NF- $\mathrm{NB} / \mathrm{p} 65$ may inhibit expression levels of FOXP3 in an miR-34a-dependent manner (35). The regulation of miR-146b-5p/Robo1/ NF- $\kappa \mathrm{B}$ system by FOXP3 has not been reported in acute lung injury. The present study tested whether the FOXP3mediated miR-146b-5p/Roundabout 1 (Robo1)/NF- $\kappa \mathrm{B}$ system invoked a protective effect on LPS-induced acute lung injury in mice.

We present the following article in accordance with the ARRIVE reporting checklist (available at http://dx.doi. org/10.21037/atm-20-7703).

\section{Methods}

\section{LPS-induced ALI model}

Adult male C57BL/6 (6-8-week-old) mice were purchased from the Beijing Vital River Laboratory Animal Technology Co. Ltd. In order to generate the ALI model, the mice were 
infected to $5 \mathrm{mg} / \mathrm{kg}$ of lipopolysaccharide (LPS, Escherichia coli O55:B5, Sigma, Saint Louis, MO) by intratracheal instillation to induce ALI and (36). All groups received intravenous injections on the $1^{\text {st }}, 3^{\text {rd }}$, and $5^{\text {th }}$ days after ALI modeling. Mice was divided into four group, including control group, ALI group, ALI + Ad-vector group and ALI + FOXP3 group.; Mice was divided into five group, including control group, ALI group, ALI + FOXP3 group, the ALI + miR antagomir group and ALI + miR antagomir+ FOXP3 group; Mice was divided into five group, including control group, ALI group, ALI + FOXP3 group, ALI + Robo1 group and ALI + FOXP3 + Robo1 group. All animal experiments were in light of the NIH Guide for the Care and Use of Laboratory Animals and were approved by the Sichuan Academy of Medical Sciences \& Sichuan Provincial People's Hospital.

\section{Cell culture and transfection}

We purchased 293T cells from the American Type Culture Collection (ATCC, Manassas, VA) and grew these in Eagle's Minimum Essential Medium (EMEM, Gibco) at $37{ }^{\circ} \mathrm{C}$ in a tissue culture chamber with $95 \% \mathrm{O}_{2}$ and $5 \% \mathrm{CO}_{2}$. Both cells stably transfected with the Ad vector or Ad-FOXP3 and those stably transfected with the Ad vector or ad-Robo1 were expanded in the presence of antibiotics and used for inoculation within three to four passages.

We then obtained miR-146b-5p mimics for overexpression at the miR-146b-5p level and mimics control (NC mimics) from GenePharma (Shanghai, China) and NC mimics, miR-146b-5p mimics and miR-146b-5p antagomir were then transfected into the $293 \mathrm{~T}$ cells. According to the manufacturer's instructions Lipofectamine 2000 reagent (Invitrogen; Thermo Fisher Scientific, Inc., Waltham, MA, USA) were employed to transfect NC mimics and miR146b-5p mimics.

\section{Quantitative real-time polymerase chain reaction ( $q R T$ - PCR)}

TRIzol reagent kit (Invitrogen, Beijing, China) were employed to isolate total RNA. In light of the manufacturer's protocol, PrimeScript RT reagent Kit (TakaRa, Dalian, China) were used to reverse transcription RNA. The qRT-PCR was employed the 2 SYBR Premix Ex Taq ${ }^{\text {TM }}$ II (TakaRa, Dalian, China) and relative expression levels of mRNA were calculated using the $2^{-\Delta \Delta \mathrm{Ct}}$ method. The sequences of qPCR primers were as follows: GAPDH-F:
ACACCCACTCCTCCACCTTT; GAPDH-R: TTACTCCTTGGAGGCCATGT; miR-146b5p-F: CCTGGCACTGAGAACTGAAT; miR-146b5p-R: GCACCAGAACTGAGTCCACA; Robo1-F: GCATCGCTGGAAGTAGCCATACT; Robo1-R: CATGAAATGGTGGGCTCAGGAT.

\section{Western blot assay}

Proteins were isolated from lung tissue using a radioimmunoprecipitation assay (RIPA) lysis buffer (Beyotime Institute of Biotechnology, Shanghai, China) according to the manufacturer's protocol. A bicinchoninic acid (BCA) Protein Assay Kit (Beyotime Institute of Biotechnology, Shanghai, China) was employed to detect the protein concentrations, and the proteins were separated using $10 \%$ dodecyl sulfate-polyacrylamide gel electrophoresis (SDSPAGE). Subsequently, the proteins were transferred onto polyvinylidene difluoride (PVDF) membranes (Merck Millipore, Germany) by electro-blotting. Next, the membranes were blocked with $5 \%$ skim milk in Tris Buffered Saline Tween (TBST, Shanghai Biyuntian Biological Co., Ltd., Shanghai) for $1 \mathrm{~h}$, and incubated overnight at $4{ }^{\circ} \mathrm{C}$ with primary antibody rabbit anti-FOXP3 $(1: 1,000, \# 12632$, cell signaling), anti-Robo1 (1:1,000, ab7972, Abcam) and p-p65 (1:1,000, \#3033, cell signaling). The membranes were subsequently incubated with corresponding antibodies conjugated to horseradish peroxidase (HRP) for $1 \mathrm{~h}$ at room temperature. GAPDH was employed as the internal control to standardize sample loading. The band densities were determined and analyzed using an automatic digital gel image analysis system (Bio-Rad CFX-96, CA, USA).

\section{Dual luciferase reporter assay}

The 293T cells were transfected with miR-146b-5p mimic then co-transfected with Robo1 wild-type reporter vector (Robo1-Wt) or Robo1 mutation of seeded sequence (Robo1-Mut) using Lipofectamine 2000. The dualluciferase reporter assay system (Promega Corporation, Madison, WI, USA) was employed to analyze the luciferase assay, following $48 \mathrm{~h}$ transfection.

\section{Histology}

Sodium pentobarbital were injected intraperitoneal to anesthetize the mice. Lung tissue was then removed and placed into $4 \%$ paraformaldehyde for $24 \mathrm{~h}$. After fixing 
with paraffin, tissue sections of $4 \mu \mathrm{m}$ thickness were obtained. Hematoxylin and eosin (HE) were then applied, and histopathological morphology observed under a light microscope.

\section{Flow cytometry analysis}

The FACS Aria cell sorter (BD Biosciences) were used to perform samples and the FlowJo (Tree Star, Ashland, OR) were used to analyze data analysis. Ultimately, M2 macrophages were identified as $\mathrm{CD} 11 \mathrm{~b}^{+} \mathrm{F} 4 / 80^{+} \mathrm{CD} 206^{+}$ cells and the number of M2 macrophages was calculated by multiplying the number of trypan blue-negative cells by the ratio of $\mathrm{CD} 11 \mathrm{~b}^{+} \mathrm{F} 4 / 80^{+} \mathrm{CD} 206^{+}$cells.

\section{ELISA assay}

The enzyme-linked immunosorbent assay (ELISA) kit were purchased from the Nanjing Institute of Bioengineering and was used to measure tumor necrosis factor- $\alpha$ (TNF- $\alpha$ ), interleukin 6 (IL-6) and IL-1 $\beta$ in lung tissue.

\section{Statistical analysis}

All experiments were carried out independently at least three times, and all experimental data were expressed as mean \pm standard deviation (SD). The Student's $t$ test and SPSS 19.0 software (SPSS Inc.) were used for statistical analysis between the two groups. $\mathrm{P}<0.05$ was considered statistically significant.

\section{Results}

\section{FOXP3 reduced lung pathological damage in ALI model} via regulating overexpression of $m i R-146 b-5 p$

Figure $1 A$ showed the protein expression levels of FOXP3 were notably down-regulated in the ALI model and levels of FOXP3 were down-regulated in the ALI model group of infection Ad-vector compared with the control group $(\mathrm{P}<0.05)$. However, the levels of FOXP3 were up-regulated in the ALI model group of infection Ad-FOXP3 compared with the Ad-vector group $(\mathrm{P}<0.05)$. The levels of mRNA expression miR-146b-5p were obviously decreased in the ALI model group compared with the control group (Figure $1 B, \mathrm{P}<0.05)$, but the levels of miR-146b-5 $\mathrm{p}$ were increased in the ALI model group of infection Ad-FOXP3 compared with the ALI model group $(\mathrm{P}<0.05)$. At the same time, the levels of miR-146b-5p were decreased in the ALI model group of injection miR-146b-5p antagomir contrasted with the ALI model group $(\mathrm{P}<0.05)$. Importantly, the levels of miR-146b-5p were increased in the ALI model group of both infection Ad-FOXP3 and injection miR-146b$5 \mathrm{p}$ antagomir compared with the ALI model group of injection miR-146b-5p antagomir $(\mathrm{P}<0.05)$. Compared to the control group, the ALI model group exhibited severe histopathologic injury, such as thickening of the alveolar wall, pulmonary congestion and decreased alveolar number (Figure 1C). The ALI model group of infection Ad-FOXP3 showed pulmonary congestion and the ALI model group of injection miR-146b-5p antagomir showed unclear alveolar walls, as well as a significant decrease in the number of alveoli. These results show that FOXP3 might reduce lung pathological damage in the ALI model by regulating overexpression of miR-146b-5p.

\section{FOXP3 increased alveolar clearance in the ALI model by mediating overexpression of miR-146b-5p}

As shown in Figure $2 A-E$, contrasted with the control group, the lung wet/dry weight ratio, myeloperoxidase, total cells, total protein and PMN in bronchoalveolar lavage fluid (BALF) were clearly elevated in the ALI model group $(\mathrm{P}<0.05)$. These same parameters were reduced in the ALI model group of infection Ad-FOXP3 compared with the ALI model group $(\mathrm{P}<0.05)$, following injection miR146b-5p antagomir $(\mathrm{P}<0.05)$. Of note, lung wet/dry weight ratio myeloperoxidase, total cells, total protein and PMN in BALF were also lower in the ALI model group of both infection Ad-FOXP3 and injection miR-146b-5p antagomir in comparison to the ALI model group of injection miR146b-5p antagomir $(\mathrm{P}<0.05)$. These results indicate that FOXP3 can also increase alveolar clearance in ALI model via mediating overexpression of miR-146b-5p.

\section{FOXP3 inbibited inflammatory response in BALF though mediating overexpression of miR-146b-5p}

The protein expressive levels of TNF- $\alpha$, IL-6 and IL-1 $\beta$ were clearly higher in the ALI model group than the control group (Figure $3 A-C, \mathrm{P}<0.01$ ). Nevertheless, the levels of TNF- $\alpha$, IL- 6 and IL- $1 \beta$ were inhibited in the ALI model group of infection Ad-FOXP3 compared with the ALI model group $(\mathrm{P}<0.01)$ following up-regulation in the ALI model group of injection miR-146b-5p antagomir $(\mathrm{P}<0.05)$. Interestingly, the levels of TNF- $\alpha$, IL- 6 and IL- $1 \beta$ were 


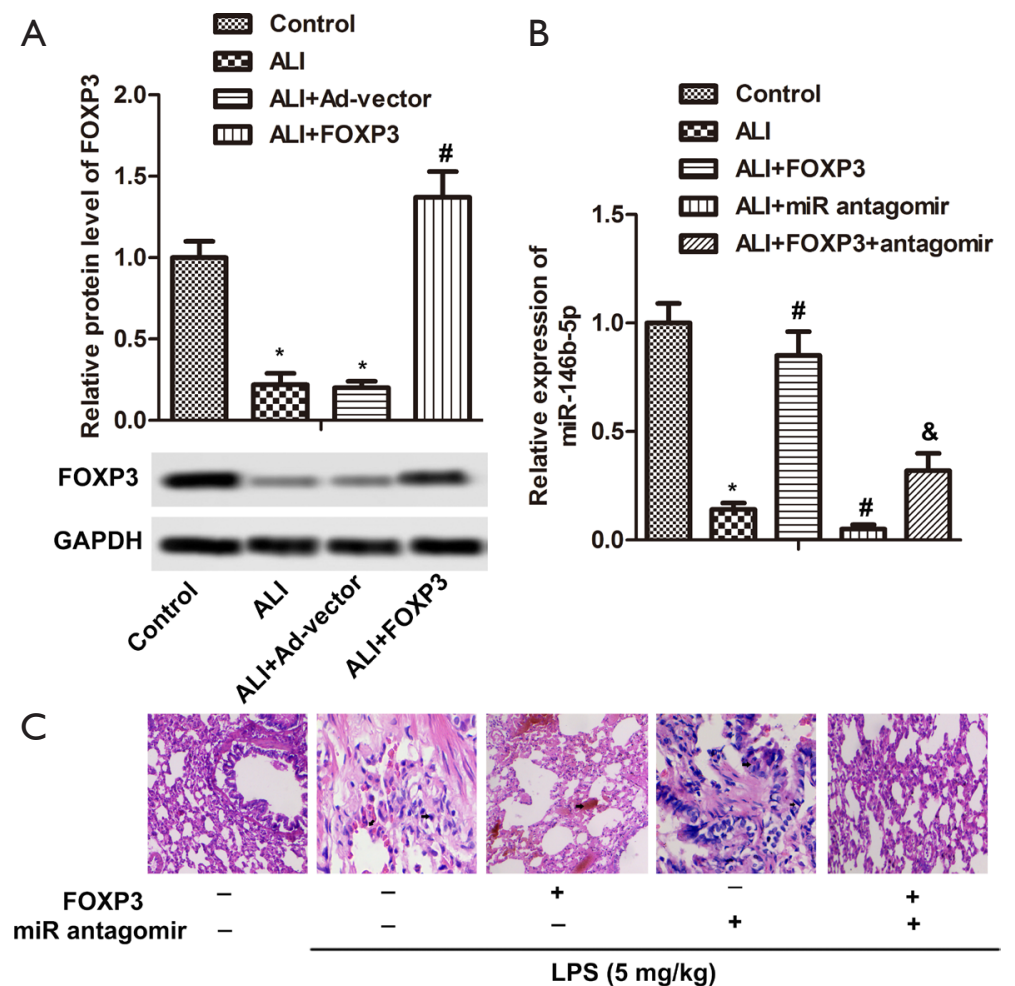

Figure 1 FOXP3 reduced lung pathological damage in the ALI model via regulating overexpression of miR-146b-5p. (A) Mice was divided into four group, including control group, ALI model group, ALI model group of infection Ad-vector and ALI model group of infection AdFOXP3. The protein levels of FOXP3 were detected by western blot in lung tissue. (B) Mice was divided control group, ALI model group, ALI model group of infection Ad-FOXP3, ALI model group of injection miR-146b-5p antagomir and ALI model group of both infection Ad-FOXP3 and injection miR-146b-5p antagomir. The mRNA levels of miR-146b-5p were detected by qRT-PCR in lung. (C) HE staining detected lung pathological damage, magnification $\times 200$. *, $\mathrm{P}<0.05$, compared with control group, * $\mathrm{P}<0.05$, compared with ALI model group, ${ }^{\&}, \mathrm{P}<0.05$, compared with ALI model group of injection miR-146b-5p antagomir. FOXP3, Forkhead box protein 3; ALI, acute lung injury; HE, hematoxylin and eosin; qRT-PCR, quantitative reverse transcription polymerase chain reaction.

suppressed in the ALI model group of both infection AdFOXP3 and injection miR-146b-5p antagomir contrasted with the ALI model group of injection miR-146b-5p antagomir $(\mathrm{P}<0.05)$. The $\mathrm{M} 2$ polarization of macrophages $\left(\mathrm{CD} 206^{+}\right)$was clearly slower in the ALI model group when compared to the control group (Figure $3 D, \mathrm{P}<0.01$ ). Nevertheless, M2 polarization of macrophages (CD206 $\left.{ }^{+}\right)$ was elevated in the ALI model group of infection Ad-FOXP3 compared with the ALI model group $(\mathrm{P}<0.01)$, following down-regulation in the ALI model group of injection miR146b-5p antagomir $(\mathrm{P}<0.05)$. The M2 polarization of macrophages $\left(\mathrm{CD} 206^{+}\right)$was promoted in the ALI model group of both infection Ad-FOXP3 and injection miR146b-5p antagomir contrasting with the ALI model group of injection miR-146b-5p antagomir $(\mathrm{P}<0.05)$. These results suggested that FOXP3 can inhibit inflammatory response in
BALF through mediating overexpression of miR-146b-5p.

\section{Determination of targeting relationship between miR- 146b-5p and Robo1}

TargetScan predicted a targeting relationship between miR-146b-5p and Robo1. The NC mimic or miR-146b$5 \mathrm{p}$ mimic was transferred into $293 \mathrm{~T}$ cells and the mRNA expressive levels of miR-146b-5p were notably increased in the miR-146b-5p mimic group compared with the NC mimic group (Figure $4 A, \mathrm{P}<0.05$ ). The dual-luciferase reporter assay was carried out on the 293T cells, showing luciferase activity was significantly inhibited in $293 \mathrm{~T}$ cells co-transfected with the wild-type Robo1 3'-UTR and miR$146 \mathrm{~b}-5 \mathrm{p}$ mimic (Figure $4 B, \mathrm{P}<0.05$ ). This result indicates that Robo1 is a potential target of miR-146b-5p (Figure 4C). 

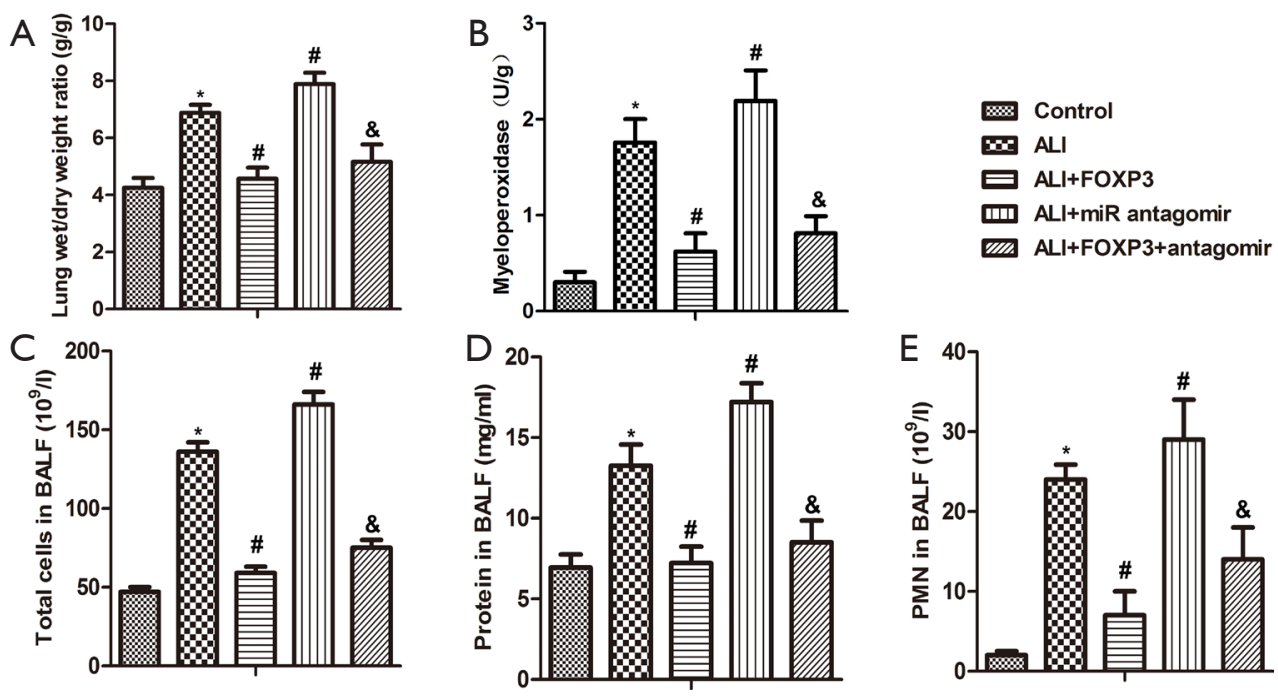

Figure 2 FOXP3 increased alveolar clearance in the ALI model via mediating overexpression of miR-146b-5p. (A) Lung wet/dry weight ratio = lung wet weigh/lung dry weight $\times 100 \%$; (B) Myeloperoxidase were detected by ELISA. (C) total cells were counted by Hemocytometer in BALF; (D) total protein was determined by the BCA method; (E) after being stained by Ray-Kee, the PMN was counted by microscope in BALF. * $\mathrm{P}<0.05$, compared with control group; ${ }^{*}, \mathrm{P}<0.05$, compared with ALI model group; ${ }^{*}, \mathrm{P}<0.05$, compared with ALI model group of injection miR-146b-5p antagomir. ELISA, enzyme-linked immunosorbent assay; BALF, bronchoalveolar lavage fluid; PMN, polymorphonuclear leukocyte; BCA, bicinchoninic acid.

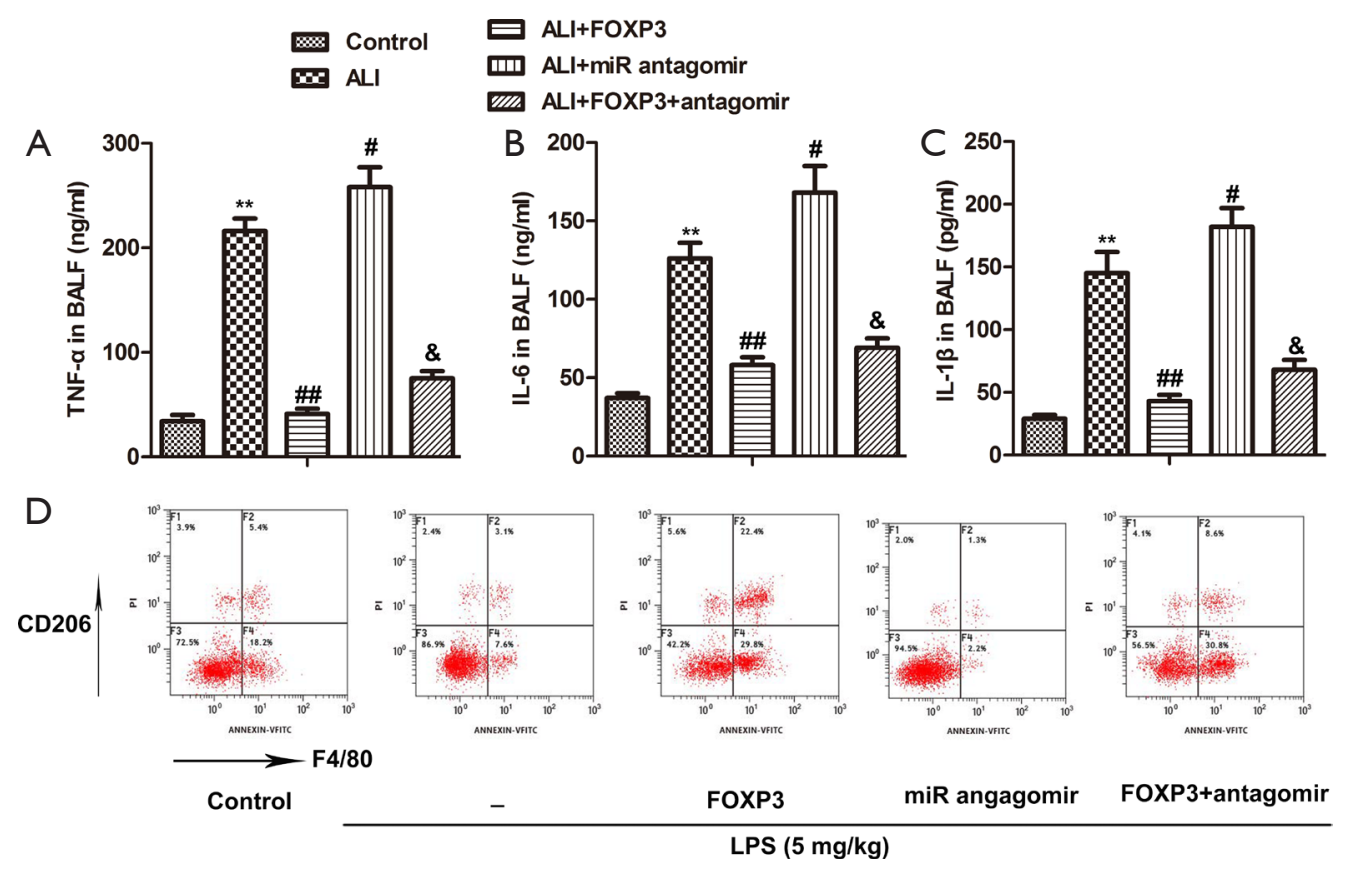

Figure 3 FOXP3 inhibited inflammatory response in BALF via mediating overexpression of miR-146. The expression levels of inflammation factors TNF- $\alpha$ (A), IL-6 (B) and IL-1 $\beta$ (C) in the BALF were detected by ELISA. (D) Representative flow cytometry plots showed theM2 phenotype $\left(\mathrm{CD} 11 \mathrm{~b}^{+} \mathrm{F} 4 / 80^{+} \mathrm{CD} 206^{+}\right){ }^{* *}, \mathrm{P}<0.01$, compared with control group; ${ }^{\#}, \mathrm{P}<0.05,{ }^{\#}, \mathrm{P}<0.01$, compared with ALI model group; ${ }^{*}$, $\mathrm{P}<0.05$, compared with ALI model group of injection miR-146b-5p antagomir. TNF- $\alpha$, tumor necrosis factor- $\alpha$; IL-6, interleukin 6 . 

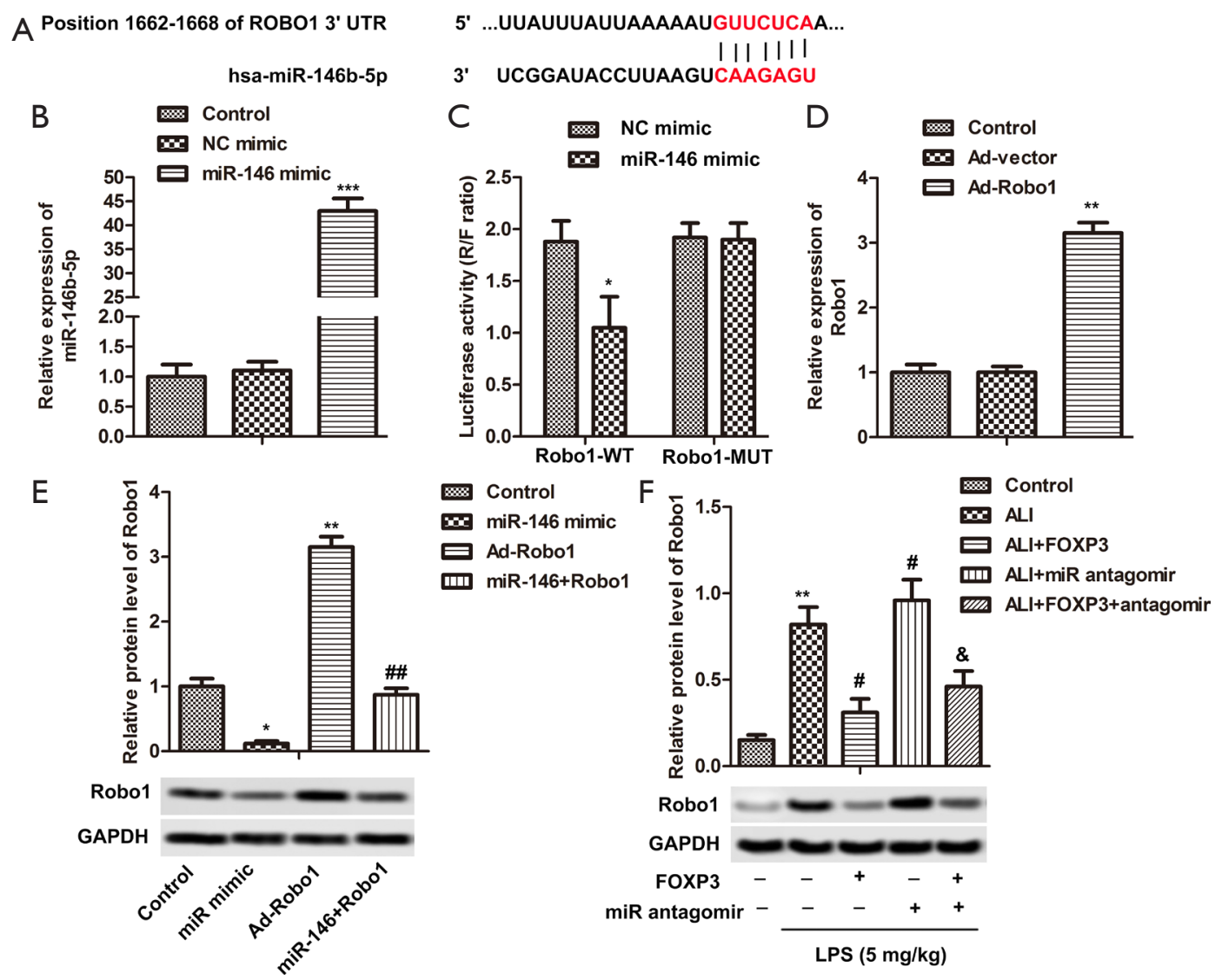

Figure 4 Determination of targeting relationship between miR-146b-5p and Robo1. (A) Predicted binding sites betweenRobo1 and miR146b-5p. (B) The mRNA expressive levels of miR-146b-5p in 293T cells were detected by RT-qPCR. (C) 293T cells were co-transfected with Robo1-Wt or Robo1-Mut and miR-146b-5p mimic, followed by the measurement of luciferase activity. (D) The mRNA expressive levels of Robo1 in 293T cells was detected by RT-qPCR. (E) The relative protein levels of Robolwere detected by western blot in the control group, miR-146b-5p mimic group, Robo1 group, miR-146b-5p mimic + Robo1 group. (F) The relative protein levels of Robo1 were detected by western blot. * $\mathrm{P}<0.05$, **, $\mathrm{P}<0.01$, ***, $\mathrm{P}<0.001$, compared with control group;, $\mathrm{P}<0.05$, compared with ALI model group; ${ }^{*}$, $\mathrm{P}<0.05$, compared with ALI model group of injection miR-146b-5p antagomir; ${ }^{\#}, \mathrm{P}<0.01$, compared with Ad-Robo1. Robo1, Roundabout 1 .

When $293 \mathrm{~T}$ cells were infected with Ad-vector or AdRobo1, the mRNA expressive levels of Robo1 were notably up-regulated in the Ad-Robo1 group compared with the Advector (Figure $4 D, \mathrm{P}<0.05$ ). It can be seen from Figure $4 E$ that protein expression levels of Robo1 are decreased in the miR-146b-5p mimic group compared with the control group $(\mathrm{P}<0.05)$, while the levels of Robol are elevated in the Ad-Robo1 group $(\mathrm{P}<0.05)$. Meaningfully, the levels of Robo1were low contrasted with the Robo1 group when miR-146b-5p and Robo1 were co-transfected with $293 \mathrm{~T}$ cells $(\mathrm{P}<0.05)$. Interestingly, contrasted with the control group, protein expression levels of Robo1 were obviously elevated in the ALI model group $(\mathrm{P}<0.05)$. At the same time, the protein expression levels of Robo1 were reduced in the ALI model group of infection Ad-FOXP3 compared with the ALI model group (Figure $4 F, \mathrm{P}<0.05$ ), following elevation in the ALI model group of injection miR-146b$5 \mathrm{p}$ antagomir $(\mathrm{P}<0.05)$. Of note, the protein expression levels of Robo1 were lower in the ALI model group of both infection Ad-FOXP3 and injection miR-146b-5p antagomir than the ALI model group of injection miR-146b-5p antagomir $(\mathrm{P}<0.05)$. These results suggest that miR-146b$5 \mathrm{p}$ can negatively regulate the expression of Robol.

\section{FOXP3 exerted a protective effect by mediating the miR- 146b-5p/Robo1/NF-кB system in ALI model}

Compared with the control group, the expressive levels of 

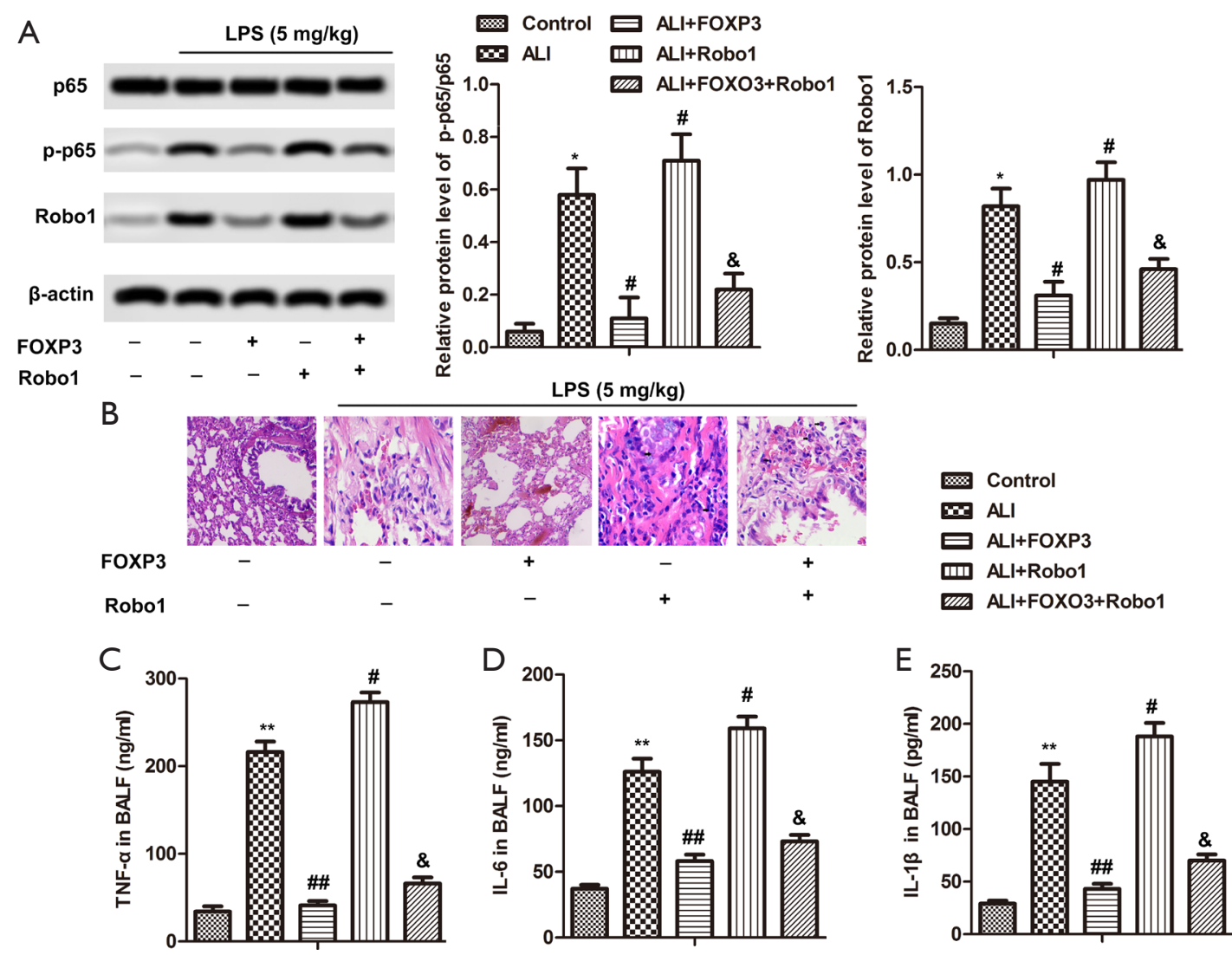

Figure 5 FOXP3 exerted a protective effect by mediating the miR-146b-5p/Robo1/NF- $\kappa$ B system in the ALI model. (A) The protein relative levels of p-65 and Robo1 were detected by western blot in the control group, ALI model group, ALI model group of infection AdFOXP3, ALI model group of infection Ad-Robo1, and ALI model group of both infection Ad-FOXP3and Ad-Robo1. (B) HE staining detected lung pathological damage. Magnification 200x. The expression levels of inflammation factors TNF- $\alpha$ (C), IL-6 (D) and IL-1 $\beta$ (E) in lung tissue were detected by ELISA. *, $\mathrm{P}<0.05$, ${ }^{*}, \mathrm{P}<0.01$, compared with control group; ${ }^{*}, \mathrm{P}<0.05,{ }^{\# \#}, \mathrm{P}<0.01$, compared with ALI model group; ${ }^{*}, \mathrm{P}<0.05$, compared with ALI model group of infection Ad-Robol.

p-65 and Robo1 protein are up-regulated markedly in the ALI model group (Figure $5 A, \mathrm{P}<0.05)$. Interestingly, the expression levels of $\mathrm{p}-65$ and Robo1 protein were repressed in the ALI model group of infection Ad-FOXP3 compared with the ALI model group $(\mathrm{P}<0.05)$ following promotion in the ALI model group of infection Ad-Robo1 $(\mathrm{P}<0.05)$. Notably, the protein expression levels of p-65 and Robo1 were inhibited in the ALI model group of co-infection Ad-FOXP3 and Ad-Robo1 in comparison to the ALI model group of infection Ad-Robo1 $(\mathrm{P}<0.05)$. As shown in Figure $5 B$, in the ALI model group of infection Ad-Robo1, the number of alveoli was significantly reduced, the alveolar walls were unclear, and inflammatory cell infiltration was seen, and a small amount of fiber exudate was seen. The protein expressive levels of TNF- $\alpha$, IL- 6 and IL-1 $\beta$ were also higher in the ALI model group than the control group
$(\mathrm{P}<0.01)$. Nevertheless, the levels of TNF- $\alpha$, IL-6 and IL$1 \beta$ were inhibited in the ALI model group of infection AdFOXP3 compared with the ALI model group (Figure 5C-E, $\mathrm{P}<0.01$ ), following up-regulation in the ALI model group of injection Ad-Robo1 $(\mathrm{P}<0.05)$. Notably, the levels of TNF- $\alpha$, IL- 6 and IL- $1 \beta$ were suppressed when the ALI model group of co-infection Ad-FOXP3 Ad-Robo1 was contrasted with the ALI model group of infection Ad-Robo1 $(\mathrm{P}<0.05)$. These results show that FOXP3 can exert a protective effect by mediating miR-146b-5p/Robo1/NF- $\mathrm{kB}$ system in the ALI model.

\section{Discussion}

In this study, FOXP3 reduced lung pathological damage and increased alveolar clearance in the ALI model, and 
inhibited the inflammatory response in BALF through regulating the overexpression of miR-146b-5p. In addition, miR-146b-5p contained complementary elements to the 3'UTR of Robo1 regions, and FOXP3 exerted a protective effect by mediating the miR-146b-5p/Robo1/NF- $\mathrm{kB}$ system in the ALI model.

FOXP3 is the transcription factor specified in the Treg cell lineage (37). Significant loss of FOXP3 and Treg function occurs under various inflammatory conditions (38). Interestingly, $\mathrm{CD}^{+} \mathrm{CD} 25^{+} \mathrm{FoxP}^{+} \mathrm{T}$ regulatory cells and CD11 $\mathrm{c}^{+}$dendritic cells protect against antibody-mediated murine transfusion-related acute lung injury via IL-10 (39). Importantly, FOXP3 expression in Tregs may be downregulated in the inflammatory alveolar microenvironment by LPS-induced ALI (9). Treg cell activation has been shown to induce the differentiation of M2-like macrophages in the myocardium, which is associated with fibroblast activation and an increased expression of monocyte/ macrophage-derived proteins, thereby promoting wound healing (40). In this study, the ALI model group exhibited severe histopathologic injury, such as thickening of the alveolar wall, pulmonary congestion and decreased alveolar number while the ALI model group of infection Ad-FOXP3 only exhibited pulmonary congestion. At the same time, the lung wet/dry weight ratio and total cells, total protein, and PMN in BALF were reduced in the ALI model group of infection Ad-FOXP3 compared with ALI the model group. In addition, the levels of TNF- $\alpha$, IL- 6 and IL- $1 \beta$ were inhibited and M2 polarization of macrophages $\mathrm{CD}_{20} 6^{+}$was elevated. These results suggest that FOXP3 can alleviate LPS-induced acute lung injury in mice.

In our study, the levels of mRNA expression miR-146b$5 \mathrm{p}$ were increased in the ALI model group of infection AdFOXP3 compared with an ALI model group. Previous research has exhibited that after FOXP3 transfection in PC3 cells, DU145cells, and LNCaP cells at 48 hours. MiR-146a/ b expression was increased dramatically (41), consistent with our results. Another report showed that the expressive levels of miR-155-5p, miR-146b-5p, and miR-142-3p were decreased in Tregs from patients with primary immune thrombocytopenia (42). Further, MiR-146 family members (miR-146a and miR-146b) were shown to coordinate and regulate germinal center $\mathrm{B}$ and follicular $\mathrm{T}$ helper (Tfh) cells; the two key arms in orchestrating protective humoral immunity (43). Most importantly, the data indicated miR$146 \mathrm{a} / \mathrm{b}$ can protect against hepatic schistosomiasis via regulating differentiation of macrophages into M2 cells (44). After injection with miR-146b-5p antagomir in the ALI model group in our study, the lung wet/dry weight ratio and total cells, total protein and PMN in BALF were elevated, and the levels of TNF- $\alpha$, IL- 6 and IL- $1 \beta$ were upregulated. In addition, the $\mathrm{M} 2$ polarization of macrophages $\left(\mathrm{CD} 206^{+}\right)$was down-regulated. Both the infection AdFOXP3 and injection miR-146b-5p antagomirin ALI model group. These results suggested that FOXP3 could increase alveolar clearance and inhibit inflammatory response in BALF though mediating overexpression of miR-146b-5p.

Previous research has shown that the protective functions of miR-146 up-regulated expressive of Sirt1, thereby blocking NF- $\kappa \mathrm{B}$ pathways (20). The low expression of miR-146b-5p promotes up-regulation of IL-17A, which then induces cell migration and invasion in $\mathrm{T}$ cell acute lymphoblastic leukemia by up-regulating matrix metalloproteinase 9 (MMP9) via NF- $\mathrm{kB}$ signaling (45). It has been shown that miR-146b-5p reduces the expression levels of TNF- $\alpha$, IL- $1 \beta$ and IL- 6 by regulating IRAK/NF- $\kappa B$ pathways in THP-1 monocytes (46). These studies have displayed that overexpression of miR-146b-5p could suppress the NF- $\mathrm{kB}$ pathway, thus reduce the inflammatory response.

Robo1 is expressed in multiple cell types, including embryonic stem cells, cardiocytes, vascular endothelial cells and mesenchymal stem cells (MSCs) (47). Robo is a protein with a single transmembrane domain (48), serving as a Slit receptor in axon guidance (49). Slit/Robo signaling can regulate a larger number of activities, such as neuronal axon guidance, angiogenesis, inflammatory cell chemotaxis, tumor cell migration and metastasis (50). The role of Slit-Robo proteins in the regulation of inflammation is controversial, with reports of both pro-inflammatory and anti-inflammatory functions. Slit2-Robo4 signaling regulates LPS-induced inflammation of endothelial cells, and LPS elevates inflammation by interfering with the expression of anti-inflammatory Slit2-Robo4 in disease states (51). Our study shows that Robo1 is a potential target of miR-146b-5p. The protein expressive levels of TNF- $\alpha$, IL- 6 and IL- $1 \beta$ were also higher in the ALI model group than the control group. Nevertheless, the levels of TNF- $\alpha$, IL-6 and IL-1 $\beta$ were inhibited in the ALI model group of infection Ad-FOXP3 compared with the ALI model group), following up-regulation in the ALI model group of injection Ad-Robo1. Notably, the levels of TNF- $\alpha$, IL-6 and IL-1 $\beta$ were suppressed when the ALI model group of co-infection Ad-FOXP3 Ad-Robo1 was contrasted with the ALI model group of infection Ad-Robo1.These results show that FOXP3 can exert a protective effect by mediating the miR-146b-5p/Robo1/NF- $\mathrm{kB}$ system in the ALI model. 
Taken together, FOXP3 induced miR-146b-5p to reduce lung damage, increase alveolar clearance, and inhibit the inflammatory response in the ALI model. In addition, Robo1, as a potential target of miR-146b-5p, could inhibit $\mathrm{NF}-\kappa \mathrm{B}$ activation, reduce lung pathological damage and inhibit inflammatory responses by mediating miR-146b-5p/ Robo1/NF-кB system in the ALI model.

\section{Acknowledgments}

Funding: None.

\section{Footnote}

Reporting Checklist: The authors have completed the ARRIVE reporting checklist. Available at http://dx.doi. org/10.21037/atm-20-7703

Data Sharing Statement: Available at http://dx.doi. org/10.21037/atm-20-7703

Conflicts of Interest: Both authors have completed the ICMJE uniform disclosure form (available at http://dx.doi. org/10.21037/atm-20-7703). The authors have no conflicts of interest to declare.

Ethical Statement: The authors are accountable for all aspects of the work in ensuring that questions related to the accuracy or integrity of any part of the work are appropriately investigated and resolved. All animal experiments were in light of the NIH Guide for the Care and Use of Laboratory Animals and were approved by the Sichuan Academy of Medical Sciences \& Sichuan Provincial People's Hospital.

Open Access Statement: This is an Open Access article distributed in accordance with the Creative Commons Attribution-NonCommercial-NoDerivs 4.0 International License (CC BY-NC-ND 4.0), which permits the noncommercial replication and distribution of the article with the strict proviso that no changes or edits are made and the original work is properly cited (including links to both the formal publication through the relevant DOI and the license). See: https://creativecommons.org/licenses/by-nc-nd/4.0/.

\section{References}

1. Ware LB, Matthay MA. The acute respiratory distress syndrome. N Engl J Med 2000;342:1334-49.

2. Goss CH, Brower RG, Hudson LD, et al. Incidence of acute lung injury in the United States. Crit Care Med 2003;31:1607-11.

3. Parekh D, Dancer RC, Thickett DR. Acute lung injury. Clin Med (Lond) 2011;11:615-8.

4. Butt Y, Kurdowska A, Allen TC. Acute Lung Injury: A Clinical and Molecular Review. Arch Pathol Lab Med 2016;140:345-50.

5. Morris PE, Glass J, Cross R, et al. Role of T-lymphocytes in the resolution of endotoxin-induced lung injury. Inflammation 1997;21:269-78.

6. Mei SH, McCarter SD, Deng Y, et al. Prevention of LPS-induced acute lung injury in mice by mesenchymal stem cells overexpressing angiopoietin 1 . PLoS Med 2007;4:e269.

7. Fontenot JD, Gavin MA, Rudensky AY. Foxp3 programs the development and function of CD4+CD25+ regulatory T cells. Nat Immunol 2003;4:330-6.

8. Mock JR, Garibaldi BT, Aggarwal NR, et al. Foxp3+ regulatory $\mathrm{T}$ cells promote lung epithelial proliferation. Mucosal Immunol 2014;7:1440-51.

9. D'Alessio FR, Tsushima K, Aggarwal NR, et al. CD4+CD25+Foxp3+ Tregs resolve experimental lung injury in mice and are present in humans with acute lung injury. J Clin Invest 2009;119:2898-913.

10. Wei Y, Schober A. MicroRNA regulation of macrophages in human pathologies. Cell Mol Life Sci 2016;73:3473-95.

11. Poy MN, Eliasson L, Krutzfeldt J, et al. A pancreatic isletspecific microRNA regulates insulin secretion. Nature 2004;432:226-30.

12. Wang R, Chen X, Xu T, et al. MiR-326 regulates cell proliferation and migration in lung cancer by targeting phox $2 \mathrm{a}$ and is regulated by HOTAIR. Am J Cancer Res 2016;6:173-86.

13. Zuo Z, Ye F, Liu Z, et al. MicroRNA-153 inhibits cell proliferation, migration, invasion and epithelialmesenchymal transition in breast cancer via direct targeting of RUNX2. Exp Ther Med 2019;17:4693-702.

14. Lee HM, Kim TS, Jo EK. MiR-146 and miR-125 in the regulation of innate immunity and inflammation. BMB Rep 2016;49:311-8.

15. Zhang L, Fu Y, Wang H, et al. Severe Fever With Thrombocytopenia Syndrome Virus-Induced Macrophage Differentiation Is Regulated by miR-146. Front Immunol 2019;10:1095.

16. Li Y, Vandenboom TG 2nd, Wang Z, et al. miR-146a suppresses invasion of pancreatic cancer cells. Cancer Res 
2010;70:1486-95.

17. Boldin MP, Taganov KD, Rao DS, et al. miR-146a is a significant brake on autoimmunity, myeloproliferation, and cancer in mice. J Exp Med 2011;208:1189-201.

18. Cai X, Lu S, Zhang Z, et al. Kaposi's sarcoma-associated herpesvirus expresses an array of viral microRNAs in latently infected cells. Proc Natl Acad Sci U S A 2005;102:5570-5.

19. Bentwich I, Avniel A, Karov Y, et al. Identification of hundreds of conserved and nonconserved human microRNAs. Nat Genet 2005;37:766-70.

20. Wang Q, Li D, Han Y, et al. MicroRNA-146 protects A549 and H1975 cells from LPS-induced apoptosis and inflammation injury. J Biosci 2017;42:637-45.

21. Liu R, Liu C, Chen D, et al. FOXP3 Controls an miR-146/NF-кB Negative Feedback Loop That Inhibits Apoptosis in Breast Cancer Cells. Cancer Res 2015;75:1703-13.

22. Li X, Zhang W, Xiao M, et al. MicroRNA-146b-5p protects oligodendrocyte precursor cells from oxygen/ glucose deprivation-induced injury through regulating Keap1/Nrf2 signaling via targeting bromodomaincontaining protein 4. Biochem Biophys Res Commun 2019;513:875-82.

23. Sheng ZX, Yao H, Cai ZY. The role of miR-146b-5p in TLR4 pathway of glomerular mesangial cells with lupus nephritis. Eur Rev Med Pharmacol Sci 2018;22:1737-43.

24. Jiang M, Lu W, Ding X, et al. p16INK4a inhibits the proliferation of osteosarcoma cells through regulating the miR-146b-5p/TRAF6 pathway.Biosci Rep 2019;39:BSR20181268.

25. Tu Z, Xiong J, Xiao R, et al. Loss of miR-146b-5p promotes $\mathrm{T}$ cell acute lymphoblastic leukemia migration and invasion via the IL-17A pathway. J Cell Biochem 2019;120:5936-48.

26. Liu J, Hou W, Guan T, et al. Slit2/Robo1 signaling is involved in angiogenesis of glomerular endothelial cells exposed to a diabetic-like environment. Angiogenesis 2018;21:237-49.

27. Ao JY, Chai ZT, Zhang YY, et al. Robol promotes angiogenesis in hepatocellular carcinoma through the Rho family of guanosine triphosphatases' signaling pathway. Tumour Biol 2015;36:8413-24.

28. Chen W, Ye L, Wen D, et al. MiR-490-5p Inhibits Hepatocellular Carcinoma Cell Proliferation, Migration and Invasion by Directly Regulating ROBO1. Pathol Oncol Res 2019;25:1-9.

29. Wang Y, Zhang S, Bao H, et al. MicroRNA-365 promotes lung carcinogenesis by downregulating the USP33/SLIT2/ ROBO1 signalling pathway.Cancer Cell Int 2018;18:64-82.

30. Covert MW, Leung TH, Gaston JE, et al. Achieving stability of lipopolysaccharide-induced NF-kappaB activation. Science 2005;309:1854-7.

31. Ghosh S, May MJ, Kopp EB. NF-kappa B and Rel proteins: evolutionarily conserved mediators of immune responses. Annu Rev Immunol 1998;16:225-60.

32. Kolb M, Margetts PJ, Anthony DC, et al. Transient expression of IL-1beta induces acute lung injury and chronic repair leading to pulmonary fibrosis. J Clin Invest 2001;107:1529-36.

33. Imanifooladi AA, Yazdani S, Nourani MR. The role of nuclear factor-kappaB in inflammatory lung disease. Inflamm Allergy Drug Targets 2010;9:197-205.

34. Zhen J, Yuan J, Fu Y, et al. IL-22 promotes Fas expression in oligodendrocytes and inhibits FOXP3 expression in $\mathrm{T}$ cells by activating the NF- $\kappa \mathrm{B}$ pathway in multiple sclerosis. Mol Immunol 2017;82:84-93.

35. Xie M, Wang J, Gong W, et al. NF-кB-driven miR34a impairs Treg/Th17 balance via targeting Foxp3. J Autoimmun 2019;102:96-113.

36. Xie W, Lu Q, Wang K, et al. miR-34b-5p inhibition attenuates lung inflammation and apoptosis in an LPSinduced acute lung injury mouse model by targeting progranulin. J Cell Physiol 2018;233:6615-31.

37. Yang $X$, Wang $W, X u J$, et al. Significant association of CD4+CD25+Foxp3+ regulatory $\mathrm{T}$ cells with clinical findings in patients with systemic lupus erythematosus. Ann Transl Med 2019;7:93.

38. Barbi J, Pardoll D, Pan F. Treg functional stability and its responsiveness to the microenvironment. Immunol Rev 2014;259:115-39.

39. Kapur R, Kim M, Aslam R, et al. T regulatory cells and dendritic cells protect against transfusion-related acute lung injury via IL-10. Blood 2017;129:2557-69.

40. Weirather J, Hofmann UD, Beyersdorf N, et al. Foxp3+ CD4+ T cells improve healing after myocardial infarction by modulating monocyte/macrophage differentiation. Circ Res 2014;115:55-67.

41. Liu R, Yi B, Wei S, et al. FOXP3-miR-146-NF-кB Axis and Therapy for Precancerous Lesions in Prostate. Cancer Res 2015;75:1714-24.

42. Zhu Y, Zhu H, Xie X, et al. MicroRNA expression profile in Treg cells in the course of primary immune thrombocytopenia. J Investig Med 2019;67:1118-24.

43. Cho S, Lee HM, Yu IS, et al. Differential cell-intrinsic regulations of germinal center $\mathrm{B}$ and $\mathrm{T}$ cells by miR-146a 
and miR-146b. Nat Commun 2018;9:2757.

44. He X, Tang R, Sun Y, et al. MicroR-146 blocks the activation of M1 macrophage by targeting signal transducer and activator of transcription 1 in hepatic schistosomiasis. EBioMedicine 2016;13:339-47.

45. Tu Z, Xiong J, Xiao R, et al. Loss of miR-146b-5p promotes $\mathrm{T}$ cell acute lymphoblastic leukemia migration and invasion via the IL-17A pathway. J Cell Biochem 2019;120:5936-48.

46. Hulsmans M, Van Dooren E, Mathieu C, et al. Decrease of miR-146b-5p in monocytes during obesity is associated with loss of the anti-inflammatory but not insulin signaling action of adiponectin. PLoS One 2012;7:e32794.

47. Yuen DA, Robinson LA. Slit2-Robo signaling: a novel regulator of vascular injury. Curr Opin Nephrol Hypertens 2013;22:445-51.

Cite this article as: Zhu J, Chen G. Protective effect of FOXP3-mediated miR-146b-5p/Robo1/NF- $\kappa$ B system on lipopolysaccharide-induced acute lung injury in mice. Ann Transl Med 2020;8(24):1651. doi: 10.21037/atm-20-7703
48. Kidd T, Brose K, Mitchell KJ, et al. Roundabout controls axon crossing of the CNS midline and defines a novel subfamily of evolutionarily conserved guidance receptors. Cell 1998;92:205-15.

49. Kidd T, Bland KS, Goodman CS. Slit is the midline repellent for the robo receptor in Drosophila. Cell 1999;96:785-94.

50. Tong M, Jun T, Nie Y, et al. The Role of the Slit/Robo Signaling Pathway. J Cancer 2019;10:2694-705.

51. Zhao H, Anand AR, Ganju RK. Slit2-Robo4 pathway modulates lipopolysaccharide-induced endothelial inflammation and its expression is dysregulated during endotoxemia. J Immunol 2014;192:385-93.

(English Language Editor: B. Draper) 